\title{
UK Parliament calls for sharing of all clinical trial data
}

$\mathrm{M}$ embers of Parliament in the United Kingdom are calling for the mandatory disclosure of all methods and results of clinical trials conducted by pharmaceutical companies.

Clinical trial information is "routinely and legally withheld from doctors and researchers by manufacturers," states a Jan. 3 report by the Public Accounts Committee. "This longstanding regulatory and cultural failure impacts on all of medicine, and undermines the ability of clinicians, researchers and patients to make informed decisions about which treatment is best."

The report focuses on one drug in particular: oseltamivir, marketed as Tamiflu by Hoffmann-La Roche. The UK Department of Health spent $£ 424$ million (\$753 million) stockpiling the antiviral medication in case of an influenza pandemic. Yet a lack of consensus remains about how well the drug works, the report states, and there is disagreement about whether regulators received all available information on Tamiflu during the licensing process.

"Tamiflu is not just any drug. It's a drug on the [World Health Organization's] essential medicines list," says Tom Jefferson, an epidemiologist with the Cochrane Collaboration in Rome, Italy. "It is stockpiled on both sides of the Atlantic, at taxpayers' expense. It is an important drug."

While reviewing the effectiveness of Tamiflu, Jefferson and colleagues discovered that $60 \%$ of the information from Hoffmann-La Roche's clinical trials of the drug was never published, he says. He spent nearly five years going back and forth with the company to gain access to that data.

Even if a pharmaceutical company does publish a clinical trial in a medical journal, that isn't enough to truly scrutinize a drug's safety and efficacy, says Jefferson. What typically ends up in a journal, he says, is a short summary of thousands of pages of information about a study designed, interpreted and described by a company to sell a product.

Instead, suggests Jefferson, all the information a company possesses about a drug should be posted on a neutral website. "Make the whole thing available. Until such time that that happens, we are not going to get around this problem."

For its part, Hoffmann-La Roche stands by the integrity and robustness of the data supporting the safety and efficacy of Tamiflu, according to an email from Lee Rammage, manager of corporate relations for Roche Canada. The company welcomes the UK report and has now provided the Cochrane Collaboration with all 77 of its completed Tamiflu studies, states the email.

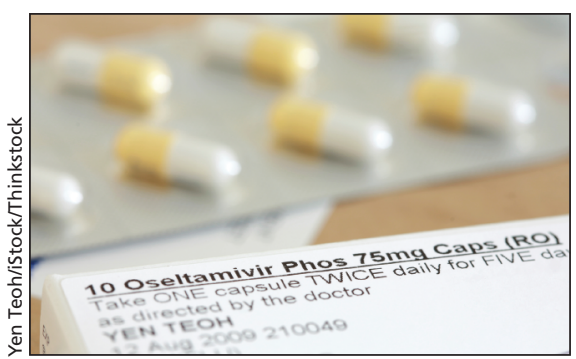

The UK Department of Health spent millions stockpiling the antiviral medication oseltamivir (Tamiflu) in case of an influenza pandemic but a lack of consensus remains about how well the drug works.

"We support the call for greater transparency in access to clinical trial results," states the email, which touts the company's data-sharing policy as exceeding industry guidelines in Europe and the United States. "This is why we expanded our policy last year to provide enhanced access to data from our clinical trials."

Calls for increased transparency have also come from organizations outside government and industry. In the US, the Institute of Medicine has a project dedicated to exploring strategies for responsible sharing of clinical trial data. It has been the topic of editorials and research papers in several prominent medical journals, including $B M J$ and PLOS ONE. In the UK, the AllTrials campaign is petitioning the public to help persuade governments and regulators to ensure that all clinical trials are registered and their full methods and results are reported.

One signee to the AllTrails campaign is GlaxoSmithKline. The pharmaceutical maker has been working to increase transparency about its research for almost a decade, according to an email statement from Dr. James Shannon, its chief medical officer. The company has an online registry where visitors can see every trial the company is running or about to start, states the email, as well as all available results whether positive or negative. "We've also launched a new website allowing scientists to request access to the very detailed, anonymized patient-level data sitting behind the results of our clinical trials," Shannon says in the email.

But some observers of the pharmaceutical industry remain skeptical of company-led initiatives to improve data transparency. They suggest that drug manufacturers, like all companies, are beholden to shareholders, which could at times conflict with their dedication to patient safety. This means selective publication - the tendency to publish positive results and withhold negative findings - may continue unless laws are created to mandate the sharing of all clinical trial data.

"There is no obligation on a manufacturer to publish the results of a study," says Dr. David Juurlink, a scientist in the evaluative clinical sciences platform at the Sunnybrook Research Institute in Toronto, Ontario. "As a physician, if I go to the literature and try to make an assessment of the best available evidence, and I only see the evidence that the companies let come to light, I'm going to make decisions that aren't sound."

According to the national association representing Canada's research-based drug companies, however, the pharmaceutical industry is already among the most regulated industries in the country. Therefore, Canadians should feel confident that their medications are safe and effective, Russell Williams, president of $\mathrm{Rx} \& \mathrm{D}$, says in an email statement. "While we cannot comment on what happens in other jurisdictions, in Canada we adhere to the very strict and comprehensive regulations guiding the disclosure of research data established and enforced by Health Canada, including the publication of clinical trial results."

\section{— Roger Collier, CMAJ}

CMAJ 2014. DOI:10.1503/cmaj.109-4697 